Original Article

\title{
Aspirin for venous thromboembolism prophylaxis after hip or knee arthroplasty: An updated meta-analysis of randomized controlled trials
}

\author{
Tarek Haykal $^{\mathrm{a}, \mathrm{b}, *}$, Babikir Kheiri ${ }^{\mathrm{a}, \mathrm{b}}$, Yazan Zayed ${ }^{\mathrm{a}, \mathrm{b}}$, Mahmoud Barbarawi ${ }^{\mathrm{a}, \mathrm{b}}$, \\ Muhammad Shah Miran $^{\mathrm{a}, \mathrm{b}}$, Adam Chahine ${ }^{\mathrm{a}, \mathrm{b}}$, Khalil Katato ${ }^{\mathrm{a}, \mathrm{c}}$, Ghassan Bachuwa, \\ ${ }^{a}$ Hurley Medical Center, Department of Internal Medicine, Flint, MI, USA \\ ${ }^{\mathrm{b}}$ Michigan State University, College of Human Medicine, East Lansing, MI, USA \\ ${ }^{\mathrm{c}}$ Genessee Hematology \& Oncology, PC, Flint, MI, USA
}

\section{A R T I C L E I N F O}

\section{Keywords:}

Aspirin

Arthroplasty

Venous thromboembolism

Thromboprophylaxis

Orthopedic surgery

Pulmonary embolism

\begin{abstract}
A B S T R A C T
Background: Patients who undergo knee or hip arthroplasty are at a significant risk of venous thromboembolism (VTE) development (pulmonary embolism and/or deep-vein thrombosis). Many different thromboprophylactic strategies have been used for the prevention of VTE in these patients with different outcomes. Therefore, our aim was to evaluate the efficacy and safety of aspirin prophylaxis when compared with placebo or anticoagulants in this population of patients.

Methods: A comprehensive electronic database search was conducted for all randomized controlled trials (RCTs) comparing the clinical outcomes of aspirin versus placebo or anticoagulants for the prevention of VTE after knee or hip arthroplasty. The primary outcome was VTE incidence. Secondary outcomes included any bleeding, major bleeding and mortality. We calculated risk ratios (RRs) and 95\% confidence intervals (CIs) using a randomeffects model at the longest possible follow-up period.

Results: We included 13 RCTs with a total of 20,115 patients with a mean age of $67.15 \pm 9.54$ and $24.39 \%$ males. Aspirin was found to be associated with a non-significantly reduced VTE events compared with other thromboprophylactic methods (RR 0.87; 95\% CI: 0.61-1.23; P = 0.43). Compared with placebo, aspirin was associated with significant reduction of VTE (RR 0.65; 95\% CI: $0.47-0.89 ; \mathrm{P}=0.008$ ). There were no significant differences in the clinical outcomes between all groups with regard to mortality (RR 0.98; 95\% CI: 0.86-1.11; $\mathrm{P}=0.72$ ), major bleeding events (RR 0.96; 95\% CI: 0.50-1.84; P = 0.91), and any bleeding events (RR: 1.09; 95\% CI: 0.82-1.44; P = 0.56).

Conclusion: Among patients who underwent knee or hip arthroplasty, aspirin prophylaxis was found to be associated with similar efficacy and safety outcomes when compared with anticoagulants. When compared with placebo, aspirin prophylaxis was associated with significantly reduced VTE and a comparable safety profile.
\end{abstract}

\section{Introduction}

Venous thromboembolism (VTE) includes both deep vein thrombosis (DVT) and pulmonary embolism, which are considered major complications following hip and knee joint-replacement surgery. Such complications carry a poor outcome with increased morbidity and mortality, and prevention of VTE is considered a cornerstone in management of these patients. ${ }^{1-3}$

Current guidelines suggest that DVT prophylaxis should be continued for at least 14 days following surgery, and may be extended to 35 days in patients at high risk for VTE. ${ }^{4}$ Pharmacologic anticoagulation is the most commonly used form of management for DVT prophylaxis in orthopedic surgeries. $^{4}$

While anticoagulants are considered effective in prevention of VTE, they carry several downfalls. An increased risk of bleeding is an inherent side effect of anticoagulation. Substantial cost of medication often limits options, especially with regard to the Novel Oral Anticoagulants (NOACs). Additionally, inconvenience of certain anticoagulants forms a significant barrier to compliance, especially regarding subcutaneous injections, thus the search for more convenient and safer strategies remains necessary. ${ }^{5}$

Aspirin is an inexpensive and widely available medication; many studies have proven its efficacy in VTE prophylaxis for patients undergoing joint replacement surgeries. ${ }^{6}$

\footnotetext{
${ }^{*}$ Corresponding author. Internal Medicine Department, Hurley Medical Center/Michigan State University, Two Hurley Plaza, 48503, USA.

E-mail address: haykalta@msu.edu (T. Haykal).
} 
Table 1

Jadad scoring of included studies.

\begin{tabular}{ll}
\hline Author & Jadad score \\
\hline Harris 1977 & $\mathbf{5}$ \\
Alfaro 1986 & $\mathbf{3}$ \\
Power 1989 & $\mathbf{5}$ \\
Gent 1996 & $\mathbf{5}$ \\
Kim 1998 & $\mathbf{3}$ \\
Rodgers 2000 & $\mathbf{5}$ \\
Gelfer 2006 & $\mathbf{3}$ \\
Westrich 2006 & $\mathbf{2}$ \\
Anderson 2013 & $\mathbf{5}$ \\
Kulshrestha 2013 & $\mathbf{5}$ \\
Jiang 2014 & $\mathbf{3}$ \\
Zou 2014 & $\mathbf{3}$ \\
Anderson 2018 & $\mathbf{5}$ \\
\hline
\end{tabular}

There have been several randomized controlled trials (RCTs) comparing various types of anticoagulants, including low-molecular-weight heparin (LMWH) and NOACs. ${ }^{7-12}$

In light of the various comparative trials, we aimed to conduct a meta-analysis evaluating the safety and efficacy of aspirin in primary prevention of VTE for patients who had undergone hip or knee-replacement surgeries while comparing it to placebo and various other types of anticoagulants.

\section{Methods}

\subsection{Data source}

We conducted our meta-analysis according to the Preferred Reporting Items for Systematic Reviews and Meta-Analyses Protocols (PRISMA-P) Statement $2015 .{ }^{13}$ We performed a comprehensive literature search of PubMed, Embase, the Cochrane Collaboration Central Register of Controlled Trials. TH, MB and YZ performed the search independently from inception to August 2018 and MSM resolved any disagreements. We used the following search terms in combination: aspirin, antiplatelet, arthroplasty, joint replacement, knee arthroplasty, hip arthroplasty, knee replacement, hip replacement,VTE, DVT, deep vein.

\subsection{Selection criteria and data extraction}

The inclusion criteria were as follows: 1) All studies must be RCTs 2) All studies must compare aspirin as thromboprophylaxis with another prophylactic modality. 3) Study subjects must be post-operative after either knee or hip arthroplasty.MB and MSM extracted data independently. Any discrepancies were resolved via consultation with TH. From each RCT we extracted trial characteristic, patient demographics, and clinical outcomes.

\subsection{Quality assessment}

The quality of the included studies was assessed independently by two authors (TH and YZ) based on the study design, number of sites, blinding to outcomes, treatment assignment generation, and proportion of follow-up completion in addition to the Jadad scoring (Table 1).

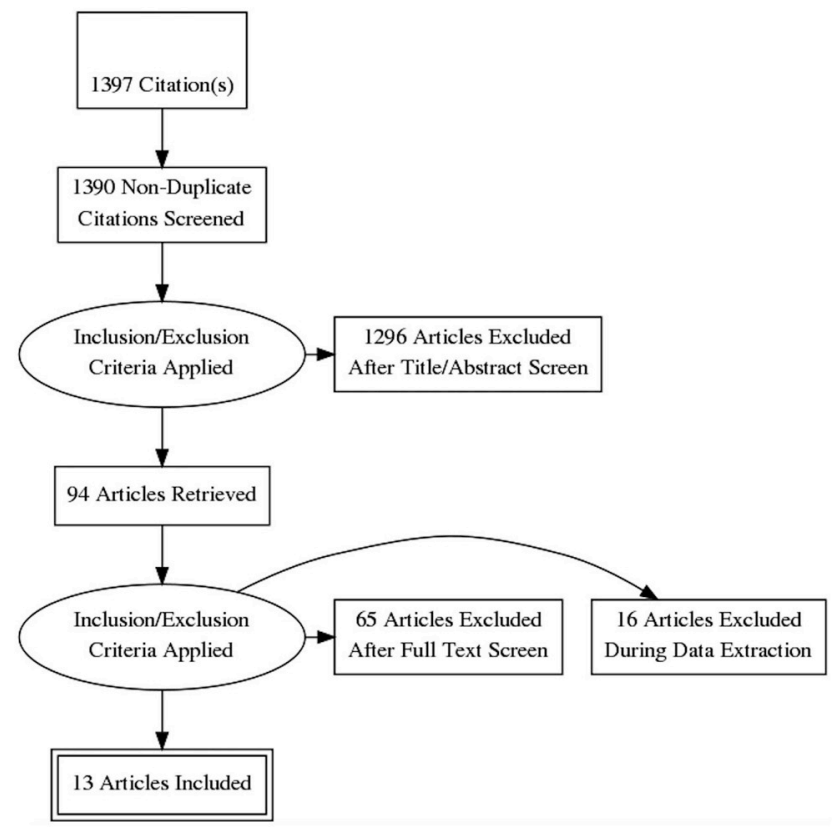

Fig. 1. The Preferred Reporting Items for Systematic Reviews and MetaAnalyses (PRISMA) flow diagram.

\subsection{Clinical outcomes}

The primary outcome was VTE. Secondary outcomes included mortality, major bleeding events, and any bleeding events. All outcomes were analyzed at the longest follow-up period.

\subsection{Statistical analysis}

We calculated the pooled risk ratios (RRs) and 95\% confidence intervals (CIs) using a random-effects model and the Mantel-Haenszel method. In addition, we calculated heterogeneity using $\mathrm{I}^{2}$ statistics. Furthermore, we constructed a funnel plot to assess for any publication bias. All data were analyzed using RevMan v5.3.

\section{Results}

Fig. 1 shows the study selection process. We included 13 RCTs with 20,115 total patients with a mean age of $67.15 \pm 9.54$ and $24.39 \%$ male patients.

Tables 2 and 3 illustrates the characteristics of the included trials and patients demographics respectively.

In these 13 studies, 20,115 patients undergoing joint arthroplasties were included. All studies were randomized controlled trials.

Of these 13 studies, 3 studies assessed VTE in both knee and hip arthroplasty, 6 studies assessed VTE in hip arthroplasty alone and 4 studies assessed VTE in knee arthroplasty alone only. Ten trials included only elective surgeries while the remaining 3 included both urgent and elective surgeries, or fracture-related urgent surgeries alone.

Aspirin dosing varied from $81 \mathrm{mg}(\mathrm{mg}$ ) daily to $1200 \mathrm{mg}$ per day, with reported thromboprophylactic regimens spanning from 14 to 35 days. Follow-up duration ranged from 4 weeks to 1 year. Other comparative thromboprophylactic measures included either new oral 
Table 2

Details of the randomized clinical trials.

\begin{tabular}{|c|c|c|c|c|c|c|}
\hline Author & Country & $\begin{array}{l}\text { Total number of } \\
\text { patients \& those } \\
\text { receiving ASA }\end{array}$ & Comparative treatment & Aspirin Dose & $\begin{array}{l}\text { Follow-up } \\
\text { period }\end{array}$ & Type of surgery \\
\hline Harris 1977 & USA & $\begin{array}{l}95 \\
\text { ASA:44 }\end{array}$ & ASA vs Placebo & $\begin{array}{l}600 \mathrm{mg} \text { bd for } 7-10 \mathrm{~d} \text { post } \\
\text { operatively }\end{array}$ & NR & $\begin{array}{l}\text { Total hip } \\
\text { replacement }\end{array}$ \\
\hline Alfaro 1986 & Spain & $\begin{array}{l}120 \\
\text { ASA: } 60\end{array}$ & $\begin{array}{l}\text { Placebo vs Low dose ASA vs High dose ASA vs } \\
\text { combination of Heparin }+ \text { dihydroergotamine } \\
\text { heparin-DHE }\end{array}$ & $\begin{array}{l}125 \mathrm{mg} \text { bd or } 500 \mathrm{mg} \text { bd, } 7 \mathrm{~d} \\
\text { post operatively }\end{array}$ & NR & $\begin{array}{l}\text { Total hip } \\
\text { replacement }\end{array}$ \\
\hline Power 1989 & Canada & $\begin{array}{l}194 \\
\text { ASA:66 }\end{array}$ & Warfarin VS ASA VS Placebo & $\begin{array}{l}650 \mathrm{mg} \text { bd for } 21 \mathrm{~d} \text { post } \\
\text { operatively or until dc }\end{array}$ & $3 \mathrm{~m}$ & $\begin{array}{l}\text { Total hip } \\
\text { replacement }\end{array}$ \\
\hline Gent 1996 & Canada & $\begin{array}{l}251 \\
\text { ASA: } 126\end{array}$ & Orgaran vs ASA & $\begin{array}{l}100 \mathrm{mg} \text { bd } 14 \mathrm{~d} \text { post } \\
\text { operatively or until dc }\end{array}$ & $3 \mathrm{~m}$ & $\begin{array}{l}\text { Total hip } \\
\text { replacement }\end{array}$ \\
\hline Kim 1998 & Korea & $\begin{array}{l}150 \\
\text { ASA:50 }\end{array}$ & Placebo vs ASA vs Dextran & $\begin{array}{l}1200 \mathrm{mg} \text { daily, } 14 \mathrm{~d} \text { post } \\
\text { operatively }\end{array}$ & NR & $\begin{array}{l}\text { Total hip } \\
\text { replacement }\end{array}$ \\
\hline Rodgers 2000 & $\begin{array}{l}\text { New } \\
\text { Zealand }\end{array}$ & $\begin{array}{l}13,356 \\
\text { ASA: } 6679\end{array}$ & ASA vs Placebo & $\begin{array}{l}160 \mathrm{mg} / \mathrm{d}, 35 \mathrm{~d} \text { post } \\
\text { operatively }\end{array}$ & 5 weeks & $\begin{array}{l}\text { Either total hip or } \\
\text { knee replacement }\end{array}$ \\
\hline Gelfer 2006 & Israel & $\begin{array}{l}121 \\
\text { ASA:61 }\end{array}$ & $\begin{array}{l}\text { WizAir continuous enhanced circulation } \\
\text { therapy }+ \text { aspirin vs Enoxaparin }\end{array}$ & $100 \mathrm{mg}$ once daily & $3 \mathrm{~m}$ & $\begin{array}{l}\text { Total hip } \\
\text { replacement }\end{array}$ \\
\hline Westrich 2006 & USA & $\begin{array}{l}275 \\
\text { ASA: } 136\end{array}$ & Enoxaparin vs ASA & $\begin{array}{l}325 \mathrm{mg} \text { bd, } 30 \mathrm{~d} \text { post } \\
\text { operatively }\end{array}$ & 6 weeks & $\begin{array}{l}\text { Total knee } \\
\text { replacement }\end{array}$ \\
\hline Anderson 2013 & Canada & $\begin{array}{l}778 \\
\text { ASA:380 }\end{array}$ & Dalteparin vs ASA & $\begin{array}{l}81 \mathrm{mg} \text { once daily between } \\
\text { post operative day } 11 \text { and } 28\end{array}$ & $90 \mathrm{~d}$ & $\begin{array}{l}\text { Either total hip or } \\
\text { knee replacement }\end{array}$ \\
\hline $\begin{array}{c}\text { Kulshrestha } \\
2013\end{array}$ & India & $\begin{array}{l}673 \\
\text { ASA:194 }\end{array}$ & routine anticoagulation vs risk stratification & $\begin{array}{l}325 \mathrm{mg} \text { bd, } 28 \mathrm{~d} \text { post } \\
\text { operatively }\end{array}$ & 1 year & $\begin{array}{l}\text { Total knee } \\
\text { replacement }\end{array}$ \\
\hline Jiang 2014 & China & $\begin{array}{l}120 \\
\text { ASA: } 60\end{array}$ & ASA + mechanical VS LMWH and rivaroxaban & $\begin{array}{l}100 \mathrm{mg} / \mathrm{d}, 14 \mathrm{~d} \text { post } \\
\text { operatively }\end{array}$ & 6 weeks & $\begin{array}{l}\text { Total knee } \\
\text { replacement }\end{array}$ \\
\hline Zou 2014 & China & $\begin{array}{l}324 \\
\text { ASA: } 110\end{array}$ & Rivaroxaban vs LMWH vs ASA & $\begin{array}{l}100 \mathrm{mg} / \mathrm{d}, 14 \mathrm{~d} \text { post } \\
\text { operatively }\end{array}$ & 4 weeks & $\begin{array}{l}\text { Total knee } \\
\text { replacement }\end{array}$ \\
\hline Anderson 2018 & Canada & $\begin{array}{l}3424 \\
\text { ASA:1707 }\end{array}$ & Rivaroxaban vs ASA & $\begin{array}{l}81 \mathrm{mg} \text { once daily starting at } \\
\text { day } 6 \text { post operatively for an } \\
\text { additional } \\
9 \text { days after total knee } \\
\text { arthroplasty or for } 30 \text { days } \\
\text { after total hip arthroplasty. }\end{array}$ & $3 \mathrm{~m}$ & $\begin{array}{l}\text { Either total hip or } \\
\text { knee replacement }\end{array}$ \\
\hline
\end{tabular}

Abbreviations: d:days; bd:twice daily; m:months; ASA:aspirin; LMWH:low molecular weight heparin; dc:discharge; NR:not reported; vs:versus;USA:United States of America.

anticoagulant, heparin, low molecular weight heparin or placebo.

Aspirin was associated with non-significantly reduced VTE compared with other thromboprophylactic strategies (RR 0.87; 95\% CI: $0.61-1.23$; $\mathrm{P}=0.43$ ). Subgroup analysis showed that aspirin was associated with significant reduction of VTE compared with placebo (RR 0.65; 95\% CI: 0.47-0.89; $\mathrm{P}=0.008$ ) (Fig. 2).

There were no significant differences in the clinical outcomes between all groups with regard to mortality (RR 0.98; 95\% CI: 0.86-1.11; $\mathrm{P}=0.72$ ), major bleeding events (RR 0.96; 95\% CI: 0.50-1.84; $\mathrm{P}=0.91$ ), and any bleeding events (RR: 1.09 ; 95\% CI: $0.82-1.44$; $\mathrm{P}=0.56$ ) (Figs. 3-5).

\section{Discussion}

In this meta-analysis of 13 RCTs, Aspirin was compared to placebo and other various anticoagulation methods including NOAC, warfarin, LMWH, and heparin. Aspirin showed a significant reduction of VTE in patients undergoing arthroplasty compared to placebo and a non-significant reduction of VTE events when compared to all other anticoagulation methods, in the exception of warfarin where Powers et al. were able to prove the superiority of warfarin (used as prophylaxis) compared to aspirin in VTE prophylaxis. However it was only one study with a small sample of only 196 patients. Therefore further larger studies are needed in the future in that regards.
Several retrospective studies have evaluated the role of aspirin in VTE prophylaxis and demonstrated the noninferiority of aspirin to anticoagulants, less bleeding events with aspirin, and less blood transfusions with aspirin. ${ }^{14-16}$ Of these retrospective studies, a large retrospective analysis of nearly 307,000 Korean patients undergoing joint replacement surgeries showed that the risk of VTE associated with aspirin was less than LMWH, similar to rivaroxaban, and slightly higher than fondaparinux, however, aspirin was associated with less blood transfusion rates and lower risk of bleeding. ${ }^{14}$

In a pooled analysis of phase III RCTs comparing the efficacy and safety of enoxaparin with rivaroxaban or dabigatran, enoxaparin held similar efficacy to dabigatran in prevention of VTE, mortality, and risk of bleeding, while rivaroxaban was associated with a $50 \%$ reduction of VTE and mortality with reduced risk of bleeding. ${ }^{17}$

Several meta-analyses have evaluated the role of primary prevention of VTE. Sahebally et al. has concluded that aspirin is effective in primary prevention of VTE in orthopedic surgeries when compared to other anticoagulants and is more effective than placebo in surgical patients. Their study involved mixed surgical populations such as orthopedic surgery fracture repairs, elective surgical procedures, and high risk non-cardiac surgeries. Additionally RCTs, meta-analyses, and prospective trials were included in their analysis. ${ }^{18}$ Another meta-analysis involving patients with a hip fracture, knee replacement, or hip replacement surgery showed the noninferiority of aspirin in comparison 


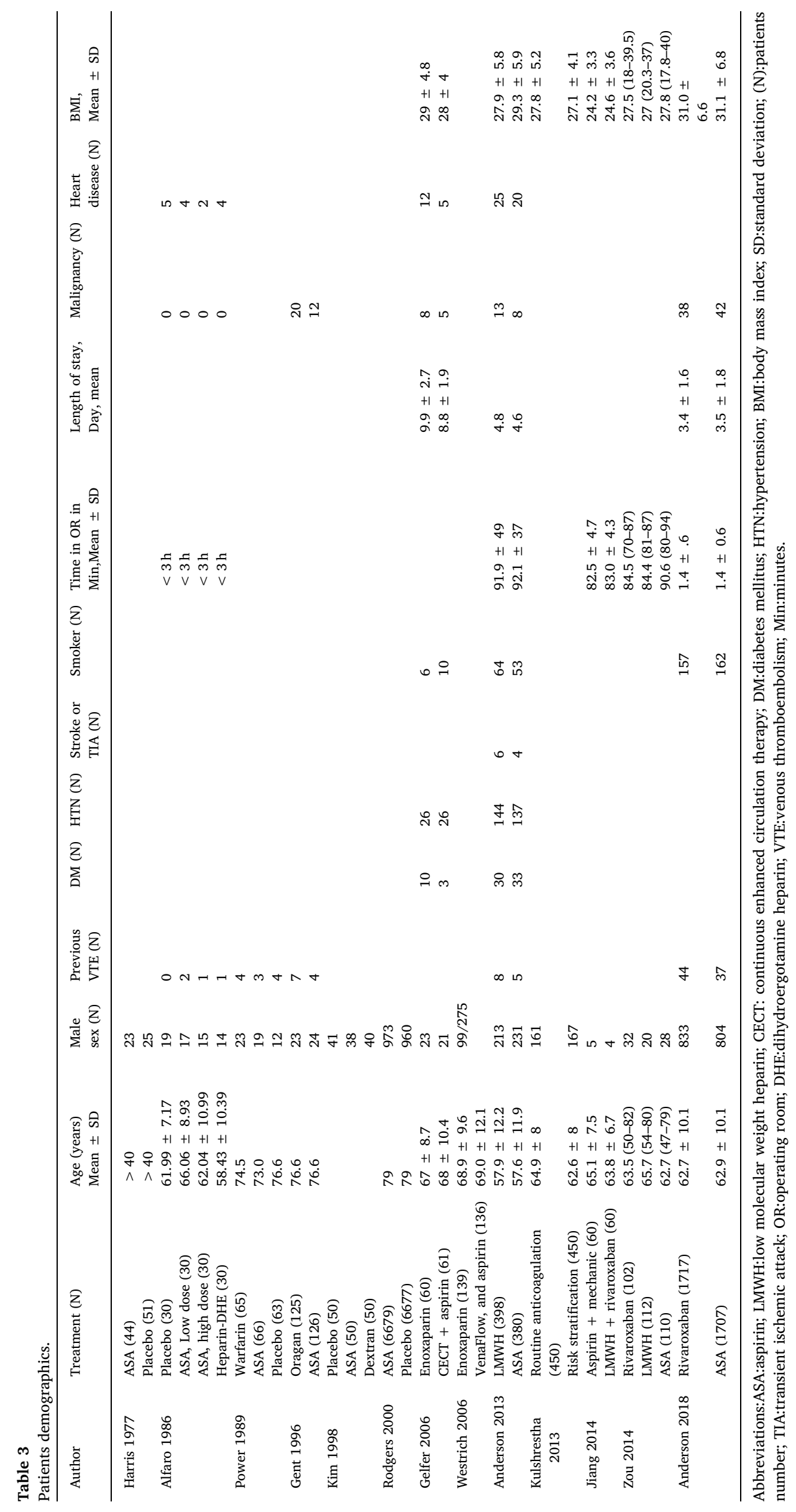






Fig. 2. Forest plot of primary outcome (venous thromboembolism).

to anticoagulants, and aspirin was associated with decreased risk of bleeding. ${ }^{19}$

An et al. performed a meta-analysis and was able to prove that aspirin, both alone and in multimodal approaches to thromboprophylaxis, confers a low rate of VTE, with a low risk of major bleeding complications. However this study included only 10 RCTs and other low quality retrospective and prospective cohort studies. ${ }^{20}$

The strengths of our meta-analysis include an extensive search of the available literature. Furthermore, we included only RCTs, which helps eliminate the likelihood of confounding biases from nonrandomized studies. However, there are several limitations in the included clinical trials. First, blinding was a major concern in the included RCTs as it was deemed not feasible in multiple studies, which could have introduced performance bias in the result. Second, due to various trial designs and protocols, there were major differences in the aspirin dosing and the different thromboprophylaxis methods used. Third, only a few clinical trials enrolled high-risk patients defined by older age or higher BMI, and few trials included patients undergoing emergency arthroplasty or patients with fractures.

\section{Conclusion}

This meta-analysis showed that among patients who underwent hip or knee arthroplasty, aspirin prophylaxis was associated with similar efficacy and safety outcomes compared with anticoagulants. Compared with placebo, aspirin prophylaxis was associated with a significantly 


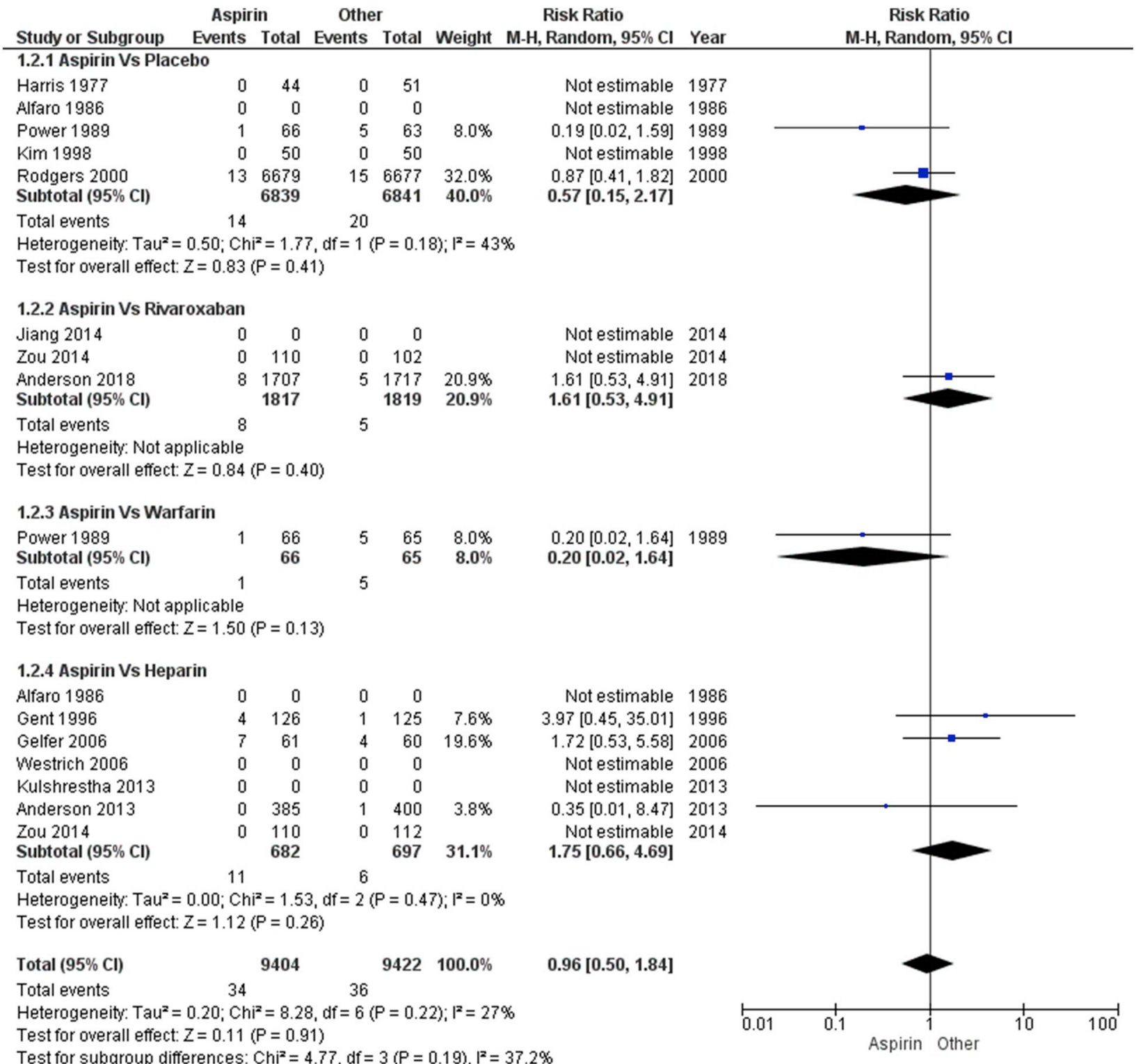

Fig. 4. Forest plot of major bleeding events. 


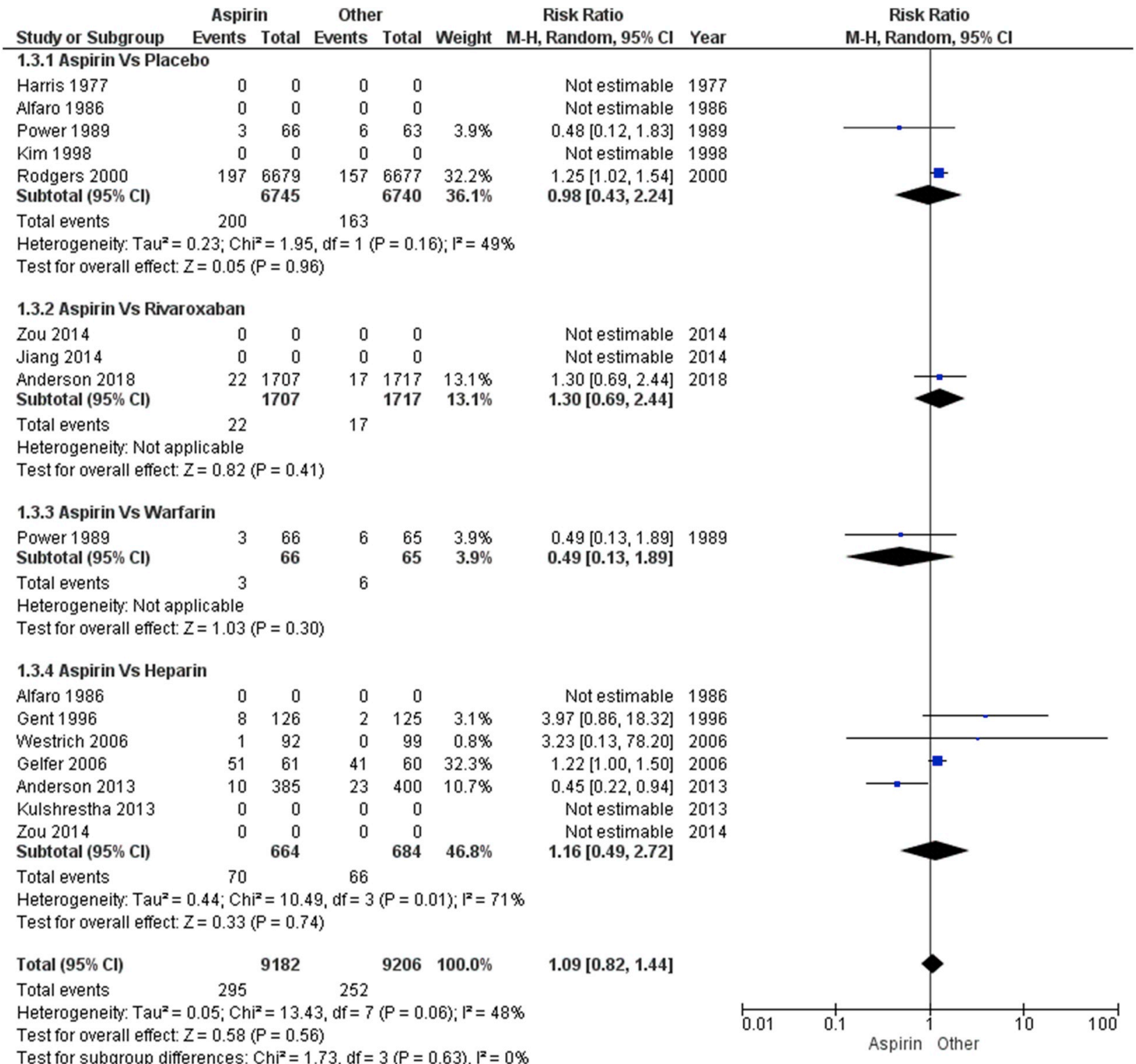

Fig. 5. Forest plot of any bleeding events.

reduced rate of VTE and a similar safety profile. Future studies are warranted to determine the exact aspirin dosing and its role in emergent arthroplasty VTE prophylaxis.

\section{Conflicts of interest}

No conflict of interest to declare.

\section{Financial disclosure}

No financial disclosure to declare.

\section{References}

1. Lieberman JR, Geerts WH. Prevention of venous thromboembolism after total hip and knee arthroplasty. J Bone Joint Surg Am. 1994;76:1239-1250.

2. Geerts WH, Bergqvist D, Pineo GF, et al. Prevention of venous thromboembolism: American College of chest Physicians evidence-based clinical practice guidelines (8th edition). Chest. 2008;133(Suppl):381S-453S.

3. Zhang LP, Tian JH, Yang K. Statins for primary prevention of venous thromboembolism. Cochrane Database Syst Rev. 2014;12 CD008203.

4. Falck-Ytter Y, Francis CW, Johanson NA, et al. Prevention of VTE in orthopedic surgery patients: antithrombotic therapy and prevention of thrombosis, 9th ed:
American College of chest Physicians evidence-based clinical practice guidelines. Chest. 2012;141(2):e278S-e325S

5. Azboy I, Barrack R, Thomas AM, Haddad FS, Parvizi J. Aspirin and the prevention of venous thromboembolism following total joint arthroplasty: commonly asked questions. Bone and Joint J. 2017;99B(11):1420-1430.

6. Olson ST, Björk I, Bock SC. Heparin and low-molecular. Trends Cardiovasc Med. 2002;99(5):198-205.

7. Eriksson BI, Borris LC, Friedman RJ, et al. Rivaroxaban versus enoxaparin for thromboprophylaxis after hip arthroplasty. N Engl J Med. 2008;358:2765-2775.

8. Kakkar AK, Brenner B, Dahl OE, et al. Extended duration rivaroxaban versus shortterm enoxaparin for the prevention of venous thromboembolism after total hip arthroplasty: a double-blind, randomized controlled trial. Lancet. 2008;372:31-39.

9. Lassen MR, Ageno W, Borris LC, et al. Rivaroxaban versus enoxaparin for thromboprophylaxis after total knee arthroplasty. N Engl J Med. 2008;358:2776-2786.

10. Turpie AG, Lassen MR, Davidson BL, et al. Rivaroxaban versus enoxaparin for thromboprophylaxis after total knee arthroplasty (RECORD4): a randomized trial. Lancet. 2009;373:1673-1680.

11. Eriksson BI, Dahl OE, Rosencher N, et al. Oral dabigatran etexilate vs subcutaneous enoxaparin for the prevention of venous thromboembolism after total knee replacement: the RE-MODEL randomized trial. J Thromb Haemost. 2007;5:2178-2185.

12. Eriksson BI, Dahl OE, Rosencher N, Kurth AA, Dijk CN, Frostick SP. Dabigatran etexilate versus enoxaparin for prevention of venous thromboembolism after total hip replacement: a randomised, double-blind, non-inferiority trial. Lancet. 2007;370:949-956.

13. Moher D, Shamseer L, Clarke M, et al. Preferred reporting items for systematic review and meta-analysis protocols (PRISMA-P) 2015 statement. Syst Rev. 2015;4:1-9.

14. Yhim HY, Lee J, Lee JY, Lee JO, Bang SM. Pharmacological thromboprophylaxis and 
its impact on venous thromboembolism following total knee and hip arthroplasty in Korea: a nationwide population-based study. PLoS One. 2017;12(05):e0178214.

15. Ogonda L, Hill J, Doran E, Dennison J, Stevenson M, Beverland D. Aspirin for thromboprophylaxis after primary lower limb arthroplasty: early thromboembolic events and 90 day mortality in 11,459 patients. Bone Joint Lett J. 2016;98-B (03):341-348.

16. Raphael IJ, Tischler EH, Huang R, Rothman RH, Hozack WJ, Parvizi J. Aspirin: an alternative for pulmonary embolism prophylaxis after arthroplasty? Clin Orthop Relat Res. 2014:472(02):482-488.

17. Huisman MV, Quinlan DJ, Dahl OE, Schulman S. Enoxaparin versus dabigatran or rivaroxaban for thromboprophylaxis after hip or knee arthroplasty: results of separate pooled analyses of phase III multicenter randomized trials. Circ Cardiovasc Qual Outcomes. 2010 Nov;3(6):652-660.

18. Sahebally SM, Healy D, Walsh SR. Aspirin in the primary prophylaxis of venous thromboembolism in surgical patients. Surgeon. 2015 Dec;13(6):348-358.

19. Drescher FS, Sirovich BE, Lee A, Morrison DH, Chiang WH. Aspirin versus anticoagulation for prevention of venous thromboembolism major lowerextremity orthopedic surgery: a systematic review and meta-analysis. J Hosp Med. 2014 Sep;9(9):579-585.

20. An VG, Phan K, Levy YD, Bruce WM. Aspirin as thromboprophylaxis in hip and knee arthroplasty: a systematic review and meta-analysis. $J$ Arthroplast. 2016;31(11):2608-2616 